\section{Deficiência auditiva referida por idosos no Município de São Paulo, Brasil: prevalência e fatores associados (Estudo SABE, 2006)}

\author{
Self-reported hearing loss among elderly \\ individuals in the city of São Paulo, Brazil: \\ prevalence and associated factors (SABE \\ Study, 2006)
}

\author{
Mariana Sodario Cruz ${ }^{1}$ \\ Maria Cristina Pereira Lima 2 \\ Jair Licio Ferreira Santos 3 \\ Yeda Aparecida de Oliveira Duarte 4 \\ Maria Lucia Lebrão 5 \\ Ana Teresa de Abreu Ramos-Cerqueira ${ }^{2}$
}

\section{Introdução}

O envelhecimento da população mundial é um fenômeno amplamente observado em países desenvolvidos e desponta como fator preponderante em países em desenvolvimento, tal como o Brasil. Dados preliminares do Censo realizado em 2010 pelo Instituto Brasileiro de Geografia e Estatística (IBGE. http://ibge.gov.br/home/es tatística/população/censo2010/default.shtm) indicaram que a população de idosos no Brasil, que era de $5 \%$ em 1960, elevou-se para 8,6\% em 2000 e atingiu aproximadamente 10,9\% em 2010. Dentre os fatores que colaboram para esse crescimento proporcional de idosos, destaca-se a melhoria das condições de vida das populações (das condições de moradia, aumento do acesso ao saneamento básico e aos serviços de saúde), avanço científico e tecnológico dos recursos ligados à saúde, migrações, dentre outros.

Apesar do aumento quantitativo dos idosos, a qualidade de vida e o amparo do Estado na manutenção dos direitos básicos desses cidadãos são, ainda, pontos incertos nas políticas e ações atuais e futuras. O campo da saúde se destaca como um dos pontos nevrálgicos nessa nova configuração etária brasileira, visto que idosos utilizam mais os serviços de saúde, se comparados às demais faixas etárias. Dessa forma, essa área se configura como uma das primeiras a remodelar sua atuação, a fim de atendê-los de forma integral, equânime e humanizada, com o objetivo 
da manutenção de sua capacidade funcional e a qualidade de vida, segundo proposto pela Política Nacional de Saúde da Pessoa Idosa ${ }^{1}$.

Entende-se por capacidade funcional a aptidão de manter as habilidades físicas e mentais necessárias para uma vida independente e autônoma ${ }^{2}$. Del Duca et al. 3 enfatizaram que o idoso que mantém sua independência e autodeterminação pode ser considerado um idoso saudável, mesmo na presença de doenças crônicas. Nessa perspectiva, o enfoque para o trabalho em saúde com tal população se distingue do de outras faixas etárias porque não se deve apenas tratar doenças agudas ou crônicas, mas também proporcionar independência e autonomia, mesmo na presença de problemas de saúde.

Uma das condições crônicas mais prevalentes na população idosa e que pode interferir em sua capacidade funcional são os prejuízos sensoriais, tal como a deficiência auditiva ${ }^{4}$. A ocorrência de deficiência auditiva aumenta progressivamente com a idade 5 devido, provavelmente, aos fatores de exposição ao longo da vida e à presbiacusia, que é o termo geral aplicado à perda auditiva relacionada à idade. A presbiacusia se caracteriza pela redução da sensibilidade auditiva e da compreensão da fala em ambientes ruidosos, lentidão do processamento central da informação acústica e localização deficiente de fontes sonoras 6 . Estima-se que a prevalência de deficiência auditiva entre idosos apresente variação de 33,7\% a 90\%, dependendo da metodologia empregada para a investigação (autorreferida ou clínica) e da faixa etária considerada 5 .

Além do avanço da idade em si, outros fatores podem contribuir para o surgimento de deficiência auditiva em idosos, ou para seu agravamento, dentre os quais se destacam: sexo masculino 7 , morbidades associadas (tais como hipertensão 8, diabetes 9 , doenças osteoarticulares 10, depressão 11 e comprometimento cognitivo 12), outras deficiências 13 , uso de medicamentos 14 , condições socioeconômicas desfavoráveis 15 , caracterizadas pela baixa renda, ocupações prévias consideradas de risco para a audição, baixa escolaridade e presença do hábito de fumar 5 .

O Brasil ainda possui poucos trabalhos relacionados ao levantamento populacional da deficiência auditiva e seus fatores associados, dentre eles se destacam os estudos realizados por Béria et al. 16 em Canoas, Rio Grande do Sul, e o levantamento da audição autorreferida em regiões do Estado de São Paulo 17. Ambas as pesquisas, de metodologia transversal, apontaram a prevalência de deficiência auditiva (clinicamente comprovada e autorreferida) de 6,8\% em Canoas e 5,2\% em São Paulo, em populações acima de quatro e 12 anos, respectivamente.
Apesar dos trabalhos iniciais, ainda há escassez de produções brasileiras na literatura especializada que ilustrem a prevalência de deficiência auditiva entre idosos, considerando uma gama maior de fatores associados, como os relativos a morbidades e estilo de vida. Com base nessa perspectiva, o objetivo do presente estudo foi estimar a prevalência de deficiência auditiva referida por idosos do Município de São Paulo no ano de 2006 e analisar seus possíveis fatores associados.

\section{Metodologia}

O presente trabalho é parte do Estudo SABE (Saúde, Bem Estar e Envelhecimento) que, em sua primeira fase, foi um multicêntrico, com a participação da Argentina, Barbados, Brasil, Chile, Cuba, México e Uruguai e teve por finalidade obter ampla quantidade de informações cujos resultados pudessem subsidiar o desenvolvimento de estudos complementares e a organização de políticas públicas para idosos. No Brasil, a população estudada foi composta pelos sujeitos residentes no Município de São Paulo.

$\mathrm{Na}$ fase um, no ano 2000, foram entrevistados 2.143 idosos, com amostra composta por dois segmentos: o primeiro, probabilístico, baseou-se em cadastro permanente de 72 setores censitários, disponível no Departamento de Epidemiologia, Faculdade de Saúde Pública, Universidade de São Paulo (FSP/USP), tomada do cadastro da Pesquisa Nacional por Amostra de Domicílios (PNAD) do ano de 1995. Esse cadastro foi composto por 263 setores censitários sorteados mediante amostragem por conglomerados, sob o critério de probabilidade proporcional ao número de domicílios e correspondeu à amostra formada por 1.568 entrevistados. O segundo segmento, composto por 575 residentes nos distritos em que se realizaram as entrevistas anteriores, correspondeu ao acréscimo efetuado para compensar a mortalidade na população de maiores de 75 anos e completar o número adequado de entrevistas. O número mínimo de domicílios sorteados no segundo estágio foi aproximado para 90 por setor censitário 18 .

Cada questionário teve peso calculado de acordo com o setor censitário correspondente (peso $=1 / \mathrm{f})$. Para os questionários realizados com indivíduos em domicílios não sorteados (faixa etária 75 anos e mais), o cálculo do peso foi realizado de acordo com a relação da população de idosos nessa faixa etária, residentes no Município de São Paulo em 1998, e o número total de idosos dessa faixa encontrado no final do estudo. Os dados para o Estudo SABE foram obtidos por 
entrevistas domiciliares, realizadas com questionários divididos em 11 blocos temáticos, aplicados por entrevistadores treinados 18 .

A partir de 2006, o Estudo SABE transformouse num estudo longitudinal que buscou estudar as alterações, com o passar do tempo, nas condições de vida e de saúde dos idosos no Município de São Paulo. Para tanto, os idosos que fizeram parte da primeira amostra foram reavaliados. $\mathrm{O}$ questionário base foi mantido, tendo sido acrescentados instrumentos complementares, inclusive no bloco de questões relativas à audição. Dos 2.143 idosos entrevistados no ano 2000, 1.028 não participaram do estudo no ano de 2006, em razão de óbito ( $n=649$ ), institucionalização $(n=11)$, mudança para outros municípios $(n=52)$, não localização $(n=139)$ e recusa $(n=177)$.

Dessa forma, a amostra considerada para o presente trabalho foi composta por 1.115 idosos com 65 anos ou mais residentes no município de São Paulo, entrevistados no ano de 2006, configurando-se um estudo transversal. Foram considerados sujeitos com deficiência auditiva referida todas as pessoas que preencheram os seguintes critérios: (1) declararam sua audição como regular, ruim ou muito ruim para a questão "Em geral, como o(a) Sr(a) diria que é a sua audição (com ou sem aparelho de audição)?"; (2) referiram ser usuárias de aparelho auditivo e/ou tiveram indicação prévia de aparelho de amplificação sonora individual.

Inquérito de saúde realizado no Canadá utilizou metodologia semelhante para o levantamento populacional da deficiência auditiva, combinando uma questão única sobre o estado auditivo com outras quatro questões sobre o uso e indicação de aparelho de amplificação sonora individual 19. Para esses autores, a complementação de questões isoladas com dados relacionados ao uso de aparelho de amplificação sonora individual aumentou a confiabilidade do instrumento, pois muitos sujeitos possuíam habilidades auditivas preservadas exatamente por conta da amplificação sonora fornecida pelo aparelho e poderiam referir que o estado auditivo atual seria bom, mesmo na presença de deficiência auditiva.

Estudou-se a associação da deficiência auditiva com os seguintes fatores: idade, sexo, cor/ etnia autorreferida, situação conjugal, arranjo domiciliar, Apgar de família, alfabetização, anos de estudo, renda, percepção de renda, grupo ocupacional, posição na ocupação, autopercepção da saúde, deficiência visual referida, uso de tabaco, morbidades referidas (diabetes, hipertensão, doenças osteoarticulares, neoplasias, doenças cardiovasculares, osteoporose, doença pulmonar obstrutiva crônica, tontura/vertigem), sintomas depressivos, provável demência, uso de medicamentos, morbidade associada à ocupação e dificuldades no desempenho de atividades de vida diária, básicas e instrumentais.

O conceito de arranjo domiciliar utilizado foi da unidade formada por uma pessoa vivendo sozinha ou por um grupo de pessoas que residem em um domicílio particular, classificando-se os arranjos em "mora sozinho ou mora acompanhado" 20. A variável "percepção de renda" foi definida com base na consideração dos idosos entrevistados sobre ter dinheiro suficiente para a cobertura das necessidades de vida diária.

O uso de tabaco foi investigado pela pesquisa sobre o hábito de fumar atualmente e/ ou ao longo da vida, sendo codificado em duas categorias: "sim", para os idosos que referiram ser fumantes atualmente ou terem sido em algum momento de sua história e "não" para os idosos que declararam nunca ter apresentado o hábito de fumar.

Para o estudo dos sintomas depressivos foi utilizada a Escala de Depressão Geriátrica GDS 21, ferramenta para a detecção de sintomas depressivos graves e leves no idoso, validada no Brasil 22. As funções cognitivas foram avaliadas pelo MEEM (Mini Exame do Estado Mental) 23, modificado e validado no Chile 24 e traduzido para o português 25. Para o presente estudo, utilizou-se ponto de corte de $12 / 13$, proposto pelo estudo de validação chileno, com o qual se obteve uma sensibilidade e especificidade de 93,8 e 93,9 respectivamente 25 , sendo o prejuízo cognitivo indicado pela pontuação igual ou inferior a 12 .

O resultado do MEEM foi utilizado como filtro para a aplicação do questionário desenvolvido por Pfeffer et al. 26. O QPAF (Pfeffer Functional Activities Questionnarie - Questionário de Pfeffer para atividades funcionais) destina-se a avaliar a autonomia funcional da pessoa em atividades da vida cotidiana. O ponto de corte utilizado foi o proposto pelos seus autores (igual ou superior a seis para indicar que o indivíduo necessita de ajuda para desenvolver atividades cotidianas denominadas instrumentais). Para efeito de análise, o sujeito que pontuava no MEEM e no QPAF foi considerado como um caso de provável demência.

O Apgar de família 27, traduzido e adaptado no Brasil 28, é um instrumento composto por cinco questões que permitem a mensuração da satisfação dos membros da família em relação a cinco componentes considerados básicos na unidade e funcionalidade de uma família: adaptação, companheirismo, desenvolvimento, afetividade e capacidade resolutiva.

As atividades básicas de vida diária envolvem as atividades de autocuidado como alimentar-se, tomar banho, vestir-se, arrumar-se, mobilizar- 
se, caminhar, controlar as necessidades fisiológicas 29 . As atividades instrumentais de vida diária remetem às atividades necessárias para o idoso para administrar o ambiente em que vive, tais como realizar compras, preparar refeições, fazer tarefas domésticas, lavar roupas, manusear dinheiro, usar o telefone, tomar medicações e utilizar os meios de transporte 30 .

Para análise das descrições das ocupações, codificaram-se as respostas com base nos grandes grupos ocupacionais propostos pela Classificação Brasileira de Ocupações 31. Foram agrupadas profissões que tivessem risco para audição similar, como presença de ruídos ambientais, similaridade de tarefas e escolaridade. Assim sendo, os novos grupos formados foram: (i) grupo 1: forças armadas, policiais e bombeiros militares, membros superiores do poder público, dirigentes de organizações de interesse público e de empresas e gerentes, profissionais das ciências e das artes; (ii) grupo 2: técnicos de nível médio; (iii) grupo 3: trabalhadores de serviços administrativos e trabalhadores dos serviços, vendedores do comércio em lojas e mercados; e (iv) grupo 4: trabalhadores agropecuários, florestais, da caça e pesca, trabalhadores da produção de bens e serviços industriais e trabalhadores de manutenção e reparação.

A posição na ocupação foi estabelecida segundo a relação de trabalho existente entre a pessoa e o empreendimento em que trabalha/ trabalhava e agrupados em categorias: "operário/trabalhador/rural”, formada pelos operários e/ou empregados com vinculo empregatício formalizado; trabalhadores rurais; trabalhadores por empreitada; trabalhadores familiares e não familiares; "autônomo", formado pelos profissionais que referiram trabalhar por conta própria; "patrão", formado por idosos que declararam ser empregadores e ou proprietários de estabelecimentos empregadores; e "outro", formado pelos sujeitos que declararam outras possibilidades de relações de trabalho.

A variável "morbidade decorrente da ocupação" teve por objetivo verificar se os idosos entrevistados referiram algum tipo de doença e ou agravo que tenha se desenvolvido exclusivamente por causa de fatores ocupacionais, com codificação de resposta "sim" para os relatos presentes e "não" para os ausentes.

A análise dos dados foi conduzida pela utilização do programa Stata 10.0 (Stata Corp., College Station, Estados Unidos), módulo survey, que permite incorporar aspectos referentes ao delineamento complexo de amostragem: estratificação não proporcional, sorteio de conglomerados e ponderação. A variável "peso", criada para ponderar os dados, foi definida pelo inverso da fração amostral e ajustada para que a amostra não apresentasse distorções no que se refere à idade e sexo.

A análise univariada foi efetuada por meio do teste de Rao-Scott, que realiza a prova de significância da associação em tabelas de dupla entrada, ajustando pelo desenho amostral, recomendado em estudos com amostras complexas 32 e a análise multivariada foi obtida pelo uso da regressão de Poisson. Foram selecionadas para integrar o modelo de regressão todas as variáveis com associação com a variável dependente, com nível de significância de $20 \%$ $(\mathrm{p}<0,20)$. Utilizou-se o procedimento retrógrado (ou passo a passo para trás) para a elaboração do modelo múltiplo, e a variável permaneceu no modelo final se $\mathrm{p}<0,05$.

O presente estudo é parte integrante do Estudo SABE, que foi aprovado pelo Comitê de Ética em Pesquisa da FSP/USP, protocolo de pesquisa número 1345, ofício COEP/83/06.

\section{Resultados}

Foram entrevistados 1.115 idosos no ano de 2006, com idades variando entre 65 e 97 anos $(\bar{\chi}=68,1$ anos e $\mathrm{DP} \pm 0,4)$. A maioria dos idosos era do sexo feminino $(61,2 \%)$, que correspondeu a um total de 701 mulheres, e a menor porcentagem $(38,8 \%)$ do masculino, representado por 414 homens.

O detalhamento dos fatores possivelmente associados à deficiência auditiva, bem como as respectivas prevalências ou distribuições encontradas em cada grupo, foram ilustradas com análises univariadas. Elas estão apresentadas nas Tabelas 1, 2, 3 e 4, divididas de acordo com seu conteúdo temático.

Foram incluídas na análise multivariada as seguintes variáveis: faixa etária, sexo, situação conjugal, alfabetização, grupo ocupacional, posição na ocupação, autopercepção da saúde, uso de tabaco, doenças osteoarticulares, doença pulmonar obstrutiva crônica, tontura/vertigem, sintomas depressivos, provável demência, deficiência visual referida, morbidades resultantes da ocupação, atividades básicas de vida diária (em sua totalidade), dificuldade para caminhar, atividades instrumentais de vida diária (total de atividades), preparar refeição quente, cuidar do dinheiro, utilizar transporte público, usar o telefone, realizar tarefas domésticas (leves e pesadas), tomar remédios.

Foi considerado desfecho deste estudo a presença ou não de deficiência auditiva referida. Assim, as variáveis que permaneceram no modelo, com $\mathrm{p}<0,05$ após a análise multivariada foram descritas na Tabela 5 . 
Prevalência de deficiência auditiva referida por idosos segundo fatores sociodemográficos. Estudo SABE, São Paulo, Brasil, 2006.

\begin{tabular}{|c|c|c|c|c|}
\hline & \multicolumn{4}{|c|}{ Deficiência auditiva referida } \\
\hline & $\mathrm{n}$ & $\%$ & IC95\% & Valor de $\mathrm{p}^{*}$ \\
\hline \multicolumn{5}{|l|}{ Faixa etária (anos) } \\
\hline $65-69$ & 121 & 25,6 & $21,0-31,0$ & $<0,001$ \\
\hline $70-74$ & 79 & 36,2 & $29,3-43,6$ & \\
\hline $75-79$ & 92 & 40,7 & $33,5-48,3$ & \\
\hline $80-84$ & 54 & 41,8 & $32,5-51,7$ & \\
\hline 85 e mais & 31 & 46,7 & $33,7-60,1$ & \\
\hline Todas & 377 & 30,4 & $27,2-33,8$ & \\
\hline \multicolumn{5}{|l|}{ Sexo } \\
\hline Masculino & 173 & 36,4 & $30,9-42,3$ & $<0,01$ \\
\hline Feminino & 204 & 26,7 & $23,0-30,8$ & \\
\hline \multicolumn{5}{|l|}{ Cor/Etnia } \\
\hline Branca & 259 & 29,8 & $25,9-34,0$ & 0,56 \\
\hline Outras & 118 & 31,9 & $26,5-37,9$ & \\
\hline \multicolumn{5}{|l|}{ Situação conjugal } \\
\hline Casado & 170 & 34,2 & $29,4-39,3$ & 0,07 \\
\hline Outros & 198 & 28,1 & $23,9-32,7$ & \\
\hline \multicolumn{5}{|l|}{ Arranjo domiciliar } \\
\hline Mora sozinho & 59 & 27,4 & $19,2-37,5$ & 0,51 \\
\hline Mora acompanhado & 316 & 30,9 & $27,3-34,7$ & \\
\hline \multicolumn{5}{|l|}{ Apgar de família } \\
\hline Boa funcionalidade & 295 & 29,6 & $26,2-33,3$ & 0,64 \\
\hline Disfunção moderada & 13 & 26,4 & $12,3-47,9$ & \\
\hline Disfunção elevada & 16 & 36,6 & $21,7-54,6$ & \\
\hline \multicolumn{5}{|l|}{ Alfabetização } \\
\hline Alfabetizado & 298 & 29,7 & $26,2-33,4$ & 0,17 \\
\hline Não alfabetizado & 79 & 34,3 & $28,4-40,8$ & \\
\hline \multicolumn{5}{|l|}{ Anos de estudo } \\
\hline Nenhum & 109 & 33,4 & $28,2-39,1$ & 0,47 \\
\hline $1-6$ & 218 & 29,8 & $25,9-34,0$ & \\
\hline Acima de 7 & 49 & 28,4 & $21,4-36,7$ & \\
\hline \multicolumn{5}{|l|}{ Renda (salários mínimos) } \\
\hline Menos de 1 & 11 & 30,6 & $16,7-49,2$ & 0,80 \\
\hline $1-2,99$ & 153 & 30,7 & $25,9-36,0$ & \\
\hline $3-4,99$ & 47 & 30,4 & $23,8-38,0$ & \\
\hline 5 ou mais & 49 & 35,2 & $26,0-45,6$ & \\
\hline \multicolumn{5}{|l|}{ Percepção de renda } \\
\hline Suficiente & 184 & 28,6 & $24,9-32,6$ & 0,25 \\
\hline Insuficiente & 181 & 32,2 & $27,1-36,7$ & \\
\hline \multicolumn{5}{|l|}{ Grupo ocupacional } \\
\hline Técnicos de nível médio & 19 & 47,7 & $30,3-65,6$ & 0,02 \\
\hline Agropecuário/Industrial/Manutenção & 160 & 34,3 & $29,3-39,7$ & \\
\hline Armadas/Executivo/Intelectual & 39 & 34,2 & $23,6-46,7$ & \\
\hline Administrativos/Serviços & 130 & 26,1 & $21,6-31,2$ & \\
\hline \multicolumn{5}{|l|}{ Posição na ocupação } \\
\hline Operário/Trabalhador/Rural & 216 & 33,5 & $28,6-38,7$ & 0,09 \\
\hline Autônomo & 89 & 33,3 & $27,7-39,3$ & \\
\hline Patrão & 14 & 21,6 & $9,9-40,8$ & \\
\hline Outros & 29 & 19,6 & $11,2-32,0$ & \\
\hline
\end{tabular}

* Teste de Rao-Scott.

IC95\%: intervalo de $95 \%$ de confiança. 
Prevalência de deficiência auditiva referida por idosos segundo autopercepção de saúde, estilo de vida, morbidades autorrelatadas e uso de medicamentos. Estudo SABE, São Paulo, Brasil, 2006.

\begin{tabular}{|c|c|c|c|c|}
\hline & \multicolumn{4}{|c|}{ Deficiência auditiva referida } \\
\hline & $\mathrm{n}$ & $\%$ & IC95\% & Valor de $p$ * \\
\hline \multicolumn{5}{|l|}{ Autopercepção da saúde } \\
\hline Muito boa ou boa & 142 & 27,3 & $23,0-32,1$ & 0,06 \\
\hline Regular, ruim ou muito ruim & 233 & 33,1 & $28,9-37,7$ & \\
\hline \multicolumn{5}{|l|}{ Deficiência visual referida } \\
\hline Não & 361 & 8,1 & $3,5-17,5$ & $<0,001$ \\
\hline Sim & 12 & 32,2 & $28,7-36,9$ & \\
\hline \multicolumn{5}{|l|}{ Uso de tabaco } \\
\hline Nunca fumou & 196 & 27,4 & $24,3-31,8$ & 0,05 \\
\hline Fuma/Já fumou & 181 & 34,3 & $28,8-40,1$ & \\
\hline \multicolumn{5}{|l|}{ Diabetes } \\
\hline Não & 292 & 29,5 & $25,8-33,4$ & 0,29 \\
\hline Sim & 83 & 33,8 & $27,1-41,6$ & \\
\hline \multicolumn{5}{|l|}{ Hipertensão } \\
\hline Não & 132 & 28,5 & $23,5-34,1$ & 0,37 \\
\hline Sim & 244 & 31,6 & $27,6-35,9$ & \\
\hline \multicolumn{5}{|l|}{ Doenças osteoarticulares } \\
\hline Não & 222 & 27,9 & $24,3-31,7$ & 0,01 \\
\hline $\operatorname{Sim}$ & 147 & 35,5 & $30,0-41,4$ & \\
\hline \multicolumn{5}{|l|}{ Neoplasias } \\
\hline Não & 354 & 30,6 & $27,4-34,0$ & 0,91 \\
\hline Sim & 23 & 29,8 & $17,5-46,0$ & \\
\hline \multicolumn{5}{|l|}{ Doenças cardiovasculares } \\
\hline Não & 336 & 29,8 & $26,3-33,6$ & 0,27 \\
\hline $\operatorname{Sim}$ & 41 & 36,9 & $26,0-49,3$ & \\
\hline \multicolumn{5}{|l|}{ Osteoporose } \\
\hline Não & 270 & 29,5 & $26,0-33,2$ & 0,22 \\
\hline Sim & 93 & 33,8 & $27,5-40,7$ & \\
\hline \multicolumn{5}{|c|}{ Doença pulmonar obstrutiva crônica } \\
\hline Não & 332 & 29,5 & $26,0-33,2$ & 0,12 \\
\hline Sim & 43 & 38,1 & $28,5-48,8$ & \\
\hline \multicolumn{5}{|l|}{ Tontura/vertigem } \\
\hline Não & 250 & 26,9 & $23,5-30,5$ & $<0,001$ \\
\hline $\operatorname{Sim}$ & 80 & 44,0 & $35,9-52,5$ & \\
\hline \multicolumn{5}{|l|}{ Sintomas depressivos ** } \\
\hline Caso & 61 & 39,3 & $28,7-50,9$ & 0,03 \\
\hline Não caso & 252 & 27,8 & $24,6-31,2$ & \\
\hline \multicolumn{5}{|l|}{ Provável demência *** } \\
\hline Caso & 72 & 38,6 & $31,0-46,7$ & 0,02 \\
\hline Não caso & 282 & 29,2 & $25,4-33,2$ & \\
\hline \multicolumn{5}{|l|}{ Faz uso de medicamentos } \\
\hline $\operatorname{Sim}$ & 351 & 30,6 & $27,3-34,3$ & 0,78 \\
\hline Não & 26 & 29,0 & $18,8-41,9$ & \\
\hline \multicolumn{5}{|c|}{ Morbidades decorrentes de ocupação } \\
\hline $\operatorname{Sim}$ & 37 & 41,9 & $29,7-55,1$ & 0,09 \\
\hline Não & 309 & 30,4 & $26,6-34,6$ & \\
\hline
\end{tabular}

* Teste de Rao-Scott;

** Segundo escores da Escala de Depressão Geriátrica - GDS;

*** Segundo achados do Mini Exame do Estado Mental (MEEM) e do Questionário de Pfeffer para Atividades Funcionais (QPAF).

IC95\%: intervalo de $95 \%$ de confiança. 
Distribuição (\%) dos idosos com e sem deficiência auditiva referida segundo dificuldades para a realização de atividades básicas de vida diária. Estudo SABE, São Paulo, Brasil, 2006.

\begin{tabular}{|c|c|c|c|c|c|}
\hline Variável & $\mathrm{n}$ & $\begin{array}{c}\text { Com deficiência } \\
\text { auditiva (\%) }\end{array}$ & $\mathrm{n}$ & $\begin{array}{c}\text { Sem deficiência } \\
\text { auditiva (\%) }\end{array}$ & Valor de $p$ * \\
\hline \multicolumn{6}{|c|}{ Dificuldade em atividades básicas de vida diária } \\
\hline Uma ou mais dificuldades & 137 & 28,1 & 192 & 20,8 & 0,02 \\
\hline Sem dificuldades & 240 & 71,9 & 532 & 79,2 & \\
\hline \multicolumn{6}{|l|}{ Quais atividades } \\
\hline \multicolumn{6}{|l|}{ Caminhar } \\
\hline Com dificuldade & 60 & 10,5 & 77 & 7,2 & 0,06 \\
\hline Sem dificuldade & 317 & 89,5 & 649 & 92,8 & \\
\hline \multicolumn{6}{|l|}{ Vestir-se } \\
\hline Com dificuldade & 69 & 12,8 & 102 & 10,6 & 0,29 \\
\hline Sem dificuldade & 308 & 87,2 & 625 & 89,4 & \\
\hline \multicolumn{6}{|l|}{ Tomar banho } \\
\hline Com dificuldade & 68 & 11,2 & 96 & 9,5 & 0,37 \\
\hline Sem dificuldade & 309 & 88,8 & 631 & 90,5 & \\
\hline \multicolumn{6}{|l|}{ Comer sozinho } \\
\hline Com dificuldade & 28 & 4,7 & 46 & 4,3 & 0,78 \\
\hline Sem dificuldade & 347 & 95,3 & 681 & 95,7 & \\
\hline \multicolumn{6}{|l|}{ Levantar da cama } \\
\hline Com dificuldade & 82 & 16,5 & 145 & 16,0 & 0,85 \\
\hline Sem dificuldade & 294 & 83,5 & 580 & 84,0 & \\
\hline \multicolumn{6}{|l|}{ Utilizar banheiro } \\
\hline Com dificuldade & 53 & 8,7 & 72 & 7,3 & 0,35 \\
\hline Sem dificuldade & 324 & 91,3 & 755 & 92,7 & \\
\hline
\end{tabular}

* Teste de Rao-Scott.

Segundo os resultados da regressão de Poisson, as variáveis relacionadas às maiores prevalências de deficiência auditiva foram: idades mais avançadas, o sexo masculino, presença de doenças osteoarticulares, relato de tontura e/ou vertigem, presença de deficiência visual referida e apresentar dificuldades na atividade de vida diária instrumental de uso do telefone.

\section{Discussão}

A prevalência total de deficiência auditiva no município de São Paulo, considerando todas as faixas etárias e ambos os sexos, foi de $30,4 \%$ (intervalo de 95\% de confiança - IC95\%: 27,2-33,8). Esses resultados assemelham-se aos achados de Cruickshanks et al. 33 , que relataram prevalência de deficiência auditiva autorreferida de 33,7\% e foram superiores aos encontrados por Coelho Filho \& Ramos 34 no Brasil (26,4\%). Desai et al. 35 , nos Estados Unidos, encontraram valores apro- ximados aos do presente estudo, tendo em conta também a deficiência auditiva autorreferida. No estudo norte-americano, cerca de $30 \%$ dos idosos com 70 anos relataram déficit auditivo, e, no presente trabalho, 36,2\% (IC95\%: 29,3-43,6). Sindhusake et al. 36 encontraram valores maiores, entretanto a faixa etária e a metodologia por eles empregada foram distintas. No referido estudo, realizado na Austrália, a prevalência de deficiência auditiva, comprovada por audiometria tonal foi de $39,4 \%$, em população acima de 55 anos. Segundo os autores, comparando-se com os achados da audição autorreferida e da clinicamente comprovada, a questão isolada "do you feel you have a hearing loss" apresentou alta sensibilidade e especificidade.

Apesar da conclusão de Sindhusake et al. 36, há trabalhos na literatura que avaliam a utilização de questões para o levantamento da deficiência auditiva como um instrumento de baixa sensibilidade e alta especificidade 37 . Desse modo, pode-se conjecturar que a prevalência 
Distribuição (\%) dos idosos com e sem deficiência auditiva referida segundo dificuldades para a realização de atividades instrumentais de vida diária. Estudo SABE, São Paulo, Brasil, 2006.

\begin{tabular}{|c|c|c|c|c|c|}
\hline Variável & $\mathbf{n}$ & $\begin{array}{c}\text { Com deficiência } \\
\text { auditiva (\%) }\end{array}$ & $\mathbf{n}$ & $\begin{array}{c}\text { Sem deficiência } \\
\text { auditiva (\%) }\end{array}$ & Valor de p * \\
\hline \multicolumn{6}{|c|}{ Dificuldade em atividades instrumentais de vida diária } \\
\hline Uma ou mais dificuldades & 55 & 79,5 & 184 & 70,3 & $<0,01$ \\
\hline Sem dificuldades & 315 & 20,5 & 537 & 29,7 & \\
\hline \multicolumn{6}{|l|}{ Quais atividades } \\
\hline \multicolumn{6}{|l|}{ Preparar refeição quente } \\
\hline Com dificuldade & 173 & 39,5 & 226 & 27,8 & $<0,001$ \\
\hline Sem dificuldade & 203 & 60,5 & 501 & 72,2 & \\
\hline \multicolumn{6}{|l|}{ Cuidar do dinheiro } \\
\hline Com dificuldade & 124 & 24,6 & 153 & 15,8 & $<0,001$ \\
\hline Sem dificuldade & 250 & 75,4 & 574 & 84,2 & \\
\hline \multicolumn{6}{|l|}{ Utilizar transporte público } \\
\hline Com dificuldade & 191 & 39,3 & 279 & 30,0 & $<0,01$ \\
\hline Sem dificuldade & 185 & 60,7 & 448 & 70,0 & \\
\hline \multicolumn{6}{|l|}{ Comprar alimentos } \\
\hline Com dificuldade & 166 & 39,7 & 297 & 37,9 & 0,61 \\
\hline Sem dificuldade & 210 & 60,3 & 430 & 62,1 & \\
\hline \multicolumn{6}{|l|}{ Usar o telefone } \\
\hline Com dificuldade & 119 & 24,7 & 138 & 14,3 & $<0,001$ \\
\hline Sem dificuldade & 256 & 75,3 & 587 & 85,7 & \\
\hline \multicolumn{6}{|l|}{ Tarefas domésticas leves } \\
\hline Com dificuldade & 192 & 45,9 & 248 & 30,9 & $<0,001$ \\
\hline Sem dificuldade & 184 & 54,1 & 476 & 69,1 & \\
\hline \multicolumn{6}{|l|}{ Tarefas domésticas pesadas } \\
\hline Com dificuldade & 281 & 69,1 & 445 & 57,7 & $<0,01$ \\
\hline Sem dificuldade & 96 & 30,9 & 281 & 42,3 & \\
\hline \multicolumn{6}{|l|}{ Tomar remédios } \\
\hline Com dificuldade & 114 & 23,3 & 153 & 15,5 & $<0,01$ \\
\hline Sem dificuldade & 263 & 76,7 & 573 & 84,5 & \\
\hline
\end{tabular}

* Teste de Rao-Scott.

de deficientes auditivos clinicamente comprovados poderia ser maior que a encontrada pelo presente estudo, fato atribuído às próprias características da deficiência auditiva. Em muitos casos, esta deficiência tem início lento e gradual, sobretudo se atribuída à presbiacusia 38 . Dessa forma, quando o idoso se percebe deficiente auditivo e relata a deficiência auditiva, ela já se encontra em estágios mais avançados, dados que fortalecem a hipótese de que a deficiência auditiva narrada tende a levantar valores de prevalência subestimados.

Os achados do Estudo SABE do ano de 2000 relativos à autopercepção da audição foram publicados por Morettin et al. 39. Entretanto, no estudo realizado no ano de 2006 , foram acrescen- tadas questões relativas ao uso e indicação de aparelho auditivo, inclusões que permitiram um delineamento mais preciso de possíveis deficiências auditivas referidas.

Com base nos dados do presente estudo, constatou-se que o número total de idosos com deficiência auditiva referida foi 377 . Contudo, o número de entrevistados para o estudo de cada fator associado, isoladamente, apresentou pequenas variações, devido às não respostas. Uma provável explicação para essas "não respostas" remeteria às dificuldades comunicativas que a deficiência auditiva pode provocar. Muitas das questões podem ter sido assinaladas como "não sabe/não respondeu" pelo não entendimento da pergunta, por dificuldade de detecção sonora ou 
Prevalência e razões de prevalência (RP), brutas e ajustadas, de deficiência auditiva referida, segundo variáveis sociodemográficas, morbidades relatadas e atividade instrumental de vida diária. Estudo SABE, São Paulo, Brasil, 2006.

\begin{tabular}{|c|c|c|c|c|c|}
\hline Variável & $\mathbf{n}$ & Prevalência (\%) & RP bruta & RP ajustada * (IC95\%) & Valor de $p$ \\
\hline \multicolumn{6}{|l|}{ Faixa etária (anos) } \\
\hline $65-69$ & 121 & 25,6 & 1,0 & 1,0 & $<0,001$ \\
\hline $70-74$ & 79 & 36,2 & 1,4 & $1,4(1,1-1,8)$ & \\
\hline $75-79$ & 92 & 40,7 & 1,6 & $1,5(1,1-2,0)$ & \\
\hline $80-84$ & 54 & 41,8 & 1,6 & $1,5(1,1-1,9)$ & \\
\hline 85 e mais & 31 & 46,7 & 1,8 & $1,5(1,0-2,4)$ & \\
\hline \multicolumn{6}{|l|}{ Sexo } \\
\hline Feminino & 204 & 26,7 & 1,0 & 1,0 & $<0,001$ \\
\hline Masculino & 173 & 36,4 & 1,4 & $1,5(1,2-1,8)$ & \\
\hline \multicolumn{6}{|c|}{ Doenças osteoarticulares } \\
\hline Não & 222 & 27,9 & 1,0 & 1,0 & $<0,01$ \\
\hline Sim & 147 & 35,5 & 1,3 & $1,3(1,1-1,6)$ & \\
\hline \multicolumn{6}{|l|}{ Tontura/Vertigem } \\
\hline Não & 250 & 26,9 & 1,0 & 1,0 & $<0,001$ \\
\hline Sim & 80 & 44,0 & 1,6 & $1,5(1,2-1,8)$ & \\
\hline \multicolumn{6}{|c|}{ Deficiência visual referida } \\
\hline Não & 361 & 8,1 & 1,0 & 1,0 & $<0,01$ \\
\hline $\operatorname{Sim}$ & 12 & 32,2 & 3,9 & $6,3(1,8-21,7)$ & \\
\hline \multicolumn{6}{|l|}{ Usar o telefone } \\
\hline Sem dificuldade & 256 & 27,8 & 1,0 & 1,0 & 0,02 \\
\hline Com dificuldade & 119 & 43,0 & 1,5 & $1,3(1,1-1,7)$ & \\
\hline
\end{tabular}

* Regressão de Poisson.

de processamento da informação acústica pelo idoso. Como segunda hipótese, pode-se supor dificuldade de entendimento pelo entrevistador da resposta atribuída, já que prejuízo no feedback auditivo do idoso pode acarretar problemas de fala, bem como distúrbios vocais 40 , situações que favoreceriam a diminuição do total de respostas consideradas em algumas questões. Apesar desse possível viés para o levantamento dos fatores associados à deficiência auditiva, especialmente os referidos, a descrição deles é de suma importância para a caracterização dessa população e para nortear ações de saúde coletiva.

Assim sendo, verifica-se que faixa etária, sexo masculino, presença de doenças osteoarticulares, relatos de tontura ou vertigem, deficiência visual referida e o relato de dificuldades para utilizar o telefone se associaram à prevalência da deficiência auditiva. Vale ressaltar que o desenho transversal dessa análise não permite a inferência da causalidade desses fatores. Porém, analisando-se os dados relativos à faixa etária e ao sexo, esta abordagem pode ser aplicada, uma vez que a presença de deficiência auditiva não deter- mina o avanço da idade e o sexo é determinado ao nascimento. $\mathrm{Na}$ análise dos demais fatores, não há possibilidade de se afirmar que a deficiência auditiva provocou maior relato de doenças osteoarticulares, tontura vertigem, deficiência visual referida e dificuldades para telefonar ou se favoreceriam o sujeito a relatar a deficiência auditiva caso tivesse tais características.

Diversos estudos identificaram a idade avançada como um dos fatores causais para a maior prevalência da deficiência auditiva 5,41, devido, entre outros fatores, ao maior tempo de exposição aos fatores prejudiciais à audição ao longo da vida e à presbiacusia propriamente dita. No Brasil, levantamento epidemiológico realizado em quatro regiões do Estado de São Paulo, incluindo a capital, verificou-se que a idade foi um fator associado à presença de deficiência auditiva, embora a prevalência fosse menor que a do presente estudo e a faixa etária considerada fosse distinta (18\% nas idades acima de 60 anos) 17.

Analisando-se as prevalências de deficiência auditiva em cada faixa etária, observou-se um aumento das taxas nas idades mais avançadas. A 
prevalência de deficiência auditiva aumenta $40 \%$ nas idades entre 70 e 74 anos e $50 \%$ nas faixas etárias acima de 75 anos, em relação às idades de referência (65-69 anos). Considerando os intervalos de confiança, esses valores são próximos aos dados de Cruickshanks et al. 33, que descreveram razão de prevalência de 1,9 de deficiência auditiva para cada aumento de cinco anos na idade de referência (43-54 anos). Esses achados corroboram a hipótese de que a presbiacusia é um fator altamente prevalente nos idosos e que a audição piora significativamente conforme aumenta a idade dos indivíduos 42 .

O sexo masculino também foi relatado como um fator associado à maior prevalência de deficiência auditiva em adultos em estudos internacionais e nacionais 5,7 . Agrawal et al. ${ }^{43}$ encontraram valores superiores ao do presente estudo, em trabalho realizado sobre a deficiência auditiva nos Estados Unidos, com razão das prevalências de deficiência auditiva entre homens e mulheres no valor de 2,4 (IC95\%: 1,7-3,5). Woodcock \& Pole 19, no Canadá, também relataram maior prevalência de deficiência auditiva referida entre homens, apesar de as prevalências, em geral, serem inferiores às encontradas em São Paulo.

A diferença entre as prevalências de deficiência auditiva entre homens e mulheres pode ser atribuída, particularmente, às diferenças de exposição ao longo da vida, visto que, provavelmente, homens podem ter desempenhado trabalhos mais insalubres ou em ambientes mais ruidosos. Todavia, fatores intrínsecos também poderiam explicar tais diferenças. Mesmo que as estruturas do sistema auditivo pareçam semelhantes para homens e mulheres ao nascimento, pequenas diferenças foram verificadas em resultados de exames de emissões otoacústicas e potenciais evocados de tronco encefálico. Consequentemente, diferenças hormonais e metabólicas também devem ser consideradas nessas análises 44 .

Considerando a literatura nacional e internacional, há carência de trabalhos populacionais que estudem a associação entre as doenças osteoarticulares e a deficiência auditiva tais como as doenças reumatológicas. Em sua maioria, esses estudos têm uma abordagem clínica, são descrição de casos ou são estudos de caso-controle 10. Em estudos mais antigos 45, chegou-se a utilizar o termo "oto-artrites" para manifestações dessa natureza (deficiência auditiva temporária percebida em crises artríticas agudas), entretanto, não foram encontrados relatos mais recentes em estudos epidemiológicos sobre essa associação.

Stone \& Francis 46 declararam que problemas auditivos podem ocorrer como complicação do curso de várias doenças reumatológicas. Desse modo, segundo os referidos autores, doenças da orelha interna imunomediada poderiam produzir, além de deficiência auditiva neurossensorial, sintomas como vertigens, tinido e sensação de plenitude auricular, ocasionando, além da diminuição da acuidade auditiva, a dificuldade de discriminação do som.

Gopinath et al. 47 em estudo epidemiológico sobre audição realizado na Austrália, o The Blue Mountains Hearing Study, verificaram que sintomas como vertigem e tontura se associaram ao zumbido, mas não à maior prevalência de deficiência auditiva. Por outro lado, Ganança et al. 48 relataram que a ocorrência de tontura, desequilíbrios e consequentes quedas são queixas muito comuns entre os idosos e poderiam estar associadas a zumbido e a dificuldades auditivas específicas, tais como: performance rebaixada em atividades de discriminação sonora, menor detecção de sons agudos e intolerância a sons intensos.

Gushikem et al. ${ }^{49}$ verificaram a ocorrência de sintomas e sinais de disfunção auditiva e vestibular em pacientes idosos com relato de tontura e detectaram que os principais fatores relacionados a essa queixa foram o zumbido, dificuldade para compreender a fala em ambientes ruidosos, hipoacusia e sensibilidade a sons intensos, em conformidade aos estudos de Ganança et al. 48. As principais queixas vestibulares identificadas foram vertigem postural, tontura não rotatória, desequilíbrio, quedas, síncopes e distúrbios neurovegetativos associados à tontura. Em relação à audição especificamente, concluíram que o principal achado na avaliação audiológica foi a deficiência auditiva neurossensorial com configuração audiométrica do tipo descendente.

Outro fator que se associou com a deficiência auditiva referida em idosos no Município de São Paulo foi deficiência visual referida. Klein et al. 50, em estudo populacional, relataram associação entre deficiência auditiva e morbidades visuais específicas, tais como maculopatia senil e catarata, sendo a deficiência auditiva considerada um fator agravante à qualidade de vida nesses pacientes.

Chia et al. ${ }^{13}$, na Austrália, relataram que sujeitos acima de 55 anos que apresentaram deficit visual relataram mais deficiência auditiva, com valores de odds ratio - OR de 1,4 (IC95\%: 1,0-2,1), se comparados aos sujeitos sem problemas visuais. O referido estudo encontrou prevalência de deficiência auditiva entre os sujeitos com deficiência visual de 65,2\%, valor superior ao do encontrado no Município de São Paulo pelo presente trabalho $(32,2 \%)$. Essas diferenças podem ser atribuídas a fatores metodológicos: no estudo australiano, foram utilizadas metodologias 
objetivas para aferição da deficiência auditiva e da deficiência visual e, no atual estudo, as morbidades foram referidas. A explicação para a maior prevalência de deficiência auditiva entre os sujeitos com déficit visual, segundo Chia et al. 13, seria que essas duas deficiências podem compartilhar fatores de risco comuns ou apresentam marcadores biológicos similares.

Dentre todas as atividades de vida diária estudadas, segundo os resultados da regressão de Poisson, somente a atividade de vida diária instrumental de usar telefone permaneceu no modelo, demonstrando que todos os prejuízos em outras atividades estavam relacionados a outros fatores, tais como idade, sexo e morbidades e não apenas à deficiência auditiva. Supunha-se que a atividade de falar ao telefone se associasse à deficiência auditiva, especialmente por conta de uma consequência dela, haja vista as características peculiares dessa atividade. Para se falar ao telefone, somente a habilidade auditiva é requerida, pois não há como realizar leitura orofacial ou utilizar algum outro mecanismo compensatório para o sucesso na atividade, a audição é o caminho único de entrada da informação.

Veiga et al. 51, em estudo sobre a satisfação de idosos e adultos deficientes auditivos usuários de aparelho de amplificação sonora individual, concluíram que o uso do telefone é a atividade mais difícil de se realizar por um sujeito com deficiência auditiva, pela dificuldade em se regular o dispositivo nessa função e as características dessa tarefa. Northern 52 também verificou que mesmo os pacientes que revelam satisfação com a prótese relatam baixos índices de melhora para o uso do telefone. Para os referidos autores, o uso do telefone é uma situação auditiva na qual as limitações tecnológicas das próteses ficam evidenciadas. Por esse motivo, as orientações de uso e manuseio da prótese auditiva devem ser reforçadas pelo treinamento de uso do telefone e o aconselhamento deve enfatizar que as dificuldades inerentes são inevitáveis, para que o paciente não crie falsas expectativas seguidas de frustração.

Outra questão a ser levantada é o possível uso dessa informação na prática clínica. Se a atividade de falar ao telefone associou-se à deficiência auditiva no presente estudo, a inclusão dessa pergunta em anamneses realizadas em idosos poderia ser um instrumento a mais para a investigação de possíveis casos de deficiência auditiva. Obviamente, não se pode transferir o risco encontrado em estudos epidemiológicos para situações individuais, ou seja, o fato de o idoso apresentar dificuldades para telefonar não indica, necessariamente, que ele apresente deficiência auditiva. Seriam necessários estudos que pes- quisassem a sensibilidade, especificidade, valor preditivo negativo e positivo de pergunta dessa natureza para a detecção de deficiência auditiva. Entretanto, por se tratar de uma questão simples e, sendo a dificuldade no uso do telefone associada à deficiência auditiva no presente estudo e, também, em estudos clínicos 52 , sugere-se aos profissionais de saúde a investigação dessa habilidade a fim de se pesquisar possível deficiência auditiva.

A deficiência auditiva em idosos também pode ser ignorada por familiares, amigos e pelo próprio sujeito acometido, uma vez que o tema ainda é pouco debatido entre a população brasileira. O levantamento dos idosos deficientes auditivos de uma comunidade é de extrema importância para a divulgação do tema e, sobretudo, para promover ações estratégicas em saúde coletiva. O planejamento de atividades de saúde pública com vistas à diminuição da prevalência dessa deficiência pode se tornar mais efetivo quando os fatores associados à deficiência são conhecidos. Segundo Veras \& Caldas 53, a informação epidemiológica deve ser valorizada por sua capacidade em prever eventos e possibilitar o diagnóstico precoce, especialmente em relação às doenças crônicas, e assim retardar o aparecimento de agravos e melhorar a qualidade de vida e abordagem terapêutica.

Mesmo que a deficiência auditiva seja inevitável, a sua detecção precoce e possível maximização do resíduo auditivo contribuem de forma ímpar para a melhoria das condições de vida do idoso, pois a audição auxilia em diversas atividades e tarefas do cotidiano, influenciando diretamente na manutenção da capacidade funcional dos idosos.

As seguintes limitações metodológicas foram detectadas na realização do presente estudo: (a) devido ao delineamento transversal não há como realizar inferências temporais ou de causalidade, salvo para sexo e idade; (b) as informações foram autorreferidas: os achados sobre deficiência auditiva bem como sobre os fatores associados podem estar superestimados ou subestimados; e (c) necessidade da compreensão auditiva para a realização dos testes específicos, como GDS e MEEM, fato que pode ter prejudicado o desempenho de idosos com deficiência auditiva nos referidos instrumentos. Segundo Liu et al. 34, o uso de questionários para o levantamento auditivo possibilita a realização de tais estudos em larga escala e facilita a coleta de dados em áreas remotas ou rurais, contudo pode gerar proporções consideráveis de falso-negativos. 


\section{Resumo}

O objetivo foi estimar a prevalência de deficiência auditiva referida numa população de idosos de São Paulo, Brasil e verificar os fatores associados, mediante pesquisa transversal, descritiva e quantitativa. A amostra foi composta por sujeitos acima de 65 anos derivada de setores censitários em dois estágios, com reposição e probabilidade proporcional à população para pessoas com 75 anos ou mais. A análise estatística foi realizada no software Stata 10, com dados ponderados, utilizando-se o teste de Rao-Scott e a regressão de Poisson do tipo stepwise backward. Foram entrevistados 1.115 idosos com prevalência de deficiência auditiva referida de 30,4\%, maior em idades mais avançadas, no sexo masculino, em sujeitos com doenças osteoarticulares referidas, queixa de vertigem elou tontura, deficiência visual referida e com dificuldades para o uso do telefone. O conhecimento da prevalência e dos fatores associados à deficiência auditiva pode auxiliar na elaboração das políticas públicas relacionadas à audição, sendo imprescindível a abordagem deste tema com a população idosa, por conta da importante ocorrência encontrada.

Perda Auditiva; Idoso; Inquéritos de Morbidade; Estudos Transversais

\section{Referências}

1. Política Nacional de Saúde da Pessoa Idosa, 2006. http://portal.saude.gov.br/portal/arquivos/pdf/ $2528 \% 20$ aprova\%20a\%20politica\%20nacional \%20de\%20saude\%20da\%20pessoa\%20idosa.pdf (acessado em 11/Fev/2011).

2. Veras RP. Envelhecimento populacional contemporâneo: demandas, desafios e inovações. Rev Saúde Pública 2009; 43:548-54.

\section{Colaboradores}

M. S. Cruz contribuiu no levantamento bibliográfico, tratamento e análise dos dados, elaboração do manuscrito e revisão final. M. C. P. Lima contribuição na concepção e planejamento, análise e interpretação dos dados, elaboração do rascunho e aprovação da versão final do manuscrito. J. L. F. Santos colaborou no tratamento dos dados, análise estatística e revisão da versão final. Y. A. O. Duarte contribuiu para o desenho do estudo, organização do banco de dados, análise e interpretação dos dados e revisão crítica da versão final. M. L. Lebrão colaborou no desenho do estudo, revisão da versão final. A. T. A. Ramos-Cerqueira contribuiu na construção do banco e análise dos dados, participação na redação dos resultados e discussão, revisão final do manuscrito.
3. Del Duca GF, Silva MC, Hallal PC. Disability relating to basic and instrumental activities of daily living among elderly subjects. Rev Saúde Pública 2009; 43:796-805.

4. Brennan M, Bally SJ. Psychosocial adaptations to dual sensory loss in middle and late adulthood. Trends Amplif 2007; 11:281-300. 
5. Cruickshanks KJ, Tweed TS, Wiley TL, Klein BEK, Klein R, Chappell R, et al. The 5-year incidence and progression of hearing loss: the epidemiology of hearing loss study. Arch Otolaryngol Head Neck Surg 2003; 129:1041-6.

6. Gates GA, Mills JH. Presbycusis. Lancet 2005; 366:1111-20.

7. Castro SS, César CLG, Carandina L, Barros MBA, Alves MCGP, Goldbaum M. Deficiência visual, auditiva e física: prevalência e fatores associados em estudo de base populacional. Cad Saúde Pública 2008; 24:1773-82.

8. Marchiori LLM, Rego Filho EA, Matsuo T. Hipertensão como fator associado à perda auditiva. Rev Bras Otorrinolaringol 2006; 72:533-40.

9. Uchida Y, Sugiura S, Ando F, Nakashima T, Shimokata H. Diabetes reduces auditory sensitivity in middle-aged listeners more than in elderly listeners: a population-based study of age-related hearing loss. Med Sci Monit 2010; 16:PH63-8.

10. Takatsu M, Higaki H, Kinoshita H, Mizushima Y, Koizuka I. Ear involvement in patients with rheumatoid arthritis. Otol Neurotol 2005; 26:755-61.

11. Loh KY, Elango S. Hearing impairment in the elderly. Med J Malaysia 2005; 60:526-9.

12. De Silva ML, McLaughlin MT, Rodrigues EJ, Broadbent JC, Gray AR, Hammond-Tooke GD. A MiniMental Status Examination for the hearing impaired. Age Ageing 2008; 37:593-5.

13. Chia EM, Mitchell P, Rochtchina E, Foran S, Golding $\mathrm{M}$, Wang JJ. Association between vision and hearing impairments and their combined effects on quality of life. Arch Ophthalmol 2006; 124: 1465-70.

14. Hippolito MA, Oliveira JA. Ototoxicidade, otoproteção e autodefesa das células ciliadas da cóclea. Medicina (Ribeiräo Preto) 2005; 38:279-89.

15. Woodcock K, Pole JD. Educational attainment, labour force status and injury: a comparison of Canadians with and without deafness and hearing loss. Int J Rehabil Res 2008; 31:297-304.

16. Béria JU, Raymann BCW, Gigante LP, Figueiredo ACL, Jotz G, Roithman R, et al. Hearing impairment and socioeconomic factors: a population-based survey of an urban locality in southern Brazil. Rev Panam Salud Pública 2007; 21:381-7.

17. Cruz MS, Oliveira LR, Carandina L, Lima MCP, César CLG, Barros MBA, et al. Prevalência de deficiência auditiva referida e causas atribuídas: um estudo de base populacional. Cad Saúde Pública 2009; 25:1123-31.

18. Lebrão ML, Laurenti R. Saúde, bem-estar e envelhecimento: o estudo SABE no município de São Paulo. Rev Bras Epidemiol 2005; 8:127-41.

19. Woodcock K, Pole JD. Health profile of deaf Canadians: analysis of the canada community health survey. Can Fam Physician 2007; 53:2140-1.

20. Medeiros M, Osório RG. Mudanças na composição dos arranjos domiciliares no Brasil - 1978 a 1998. Rev Bras Estud Popul 2000; 17:67-85.

21. Sheikh JI, Yesavage JA. Geriatric Depression Scale (GDS): recent evidence and development of a short version. Clin Gerontol 1986; 5:165-73.
22. Paradela EMP, Lourenço RA, Veras RP. Validação da escala de depressão geriátrica em um ambulatório geral. Rev Saúde Pública 2005; 39:918-23.

23. Folstein MF, Folstein SE, McHugh PR. "Mini-mental state". A practical method for grading the cognitive state of patient for the clinican. J Psychiatr Res $1975 ; 12: 189-98$.

24. Icaza MC, Albala C.. Minimental State Examination (MMSE) del estudio de dementia en Chile: análisis estadístico. Washington DC: Pan American Health Organization; 1999.

25. Bertolucci PH, Brucki SM, Campacci SR, Juliano Y. O mini-exame do estado mental em uma população geral: impacto da escolaridade. Arq Neuropsiquiatr 1994; 52:1-7.

26. Pfeffer RI, Kurosaki TT, Harrah Jr. CH, Chance JM, Filos S. Measurement of functional activities in older adults in the community. J Gerontol 1987; 37:323-9.

27. Smilkstein G. The family APGAR: a proposal for a family function test and its used by physicians. J Fam Pract 1978; 6:12-31.

28. Duarte YAO. Família: rede de suporte ou fator estressor: a ótica de idosos e cuidadores familiares [Tese de Doutorado]. São Paulo: Programa de PósGraduação em Enfermagem, Universidade de São Paulo; 2001.

29. Katz S, Ford AB, Moskowitz RW, Jackson BA, Jaffe MW. Studies of illness in the aged. The index of ADL: a standardized measure of biological ans psychosocial function. JAMA 1963; 185:914-9.

30. Lawton MP, Brody EM. Assessment of older people: self-maintaining and instrumental activities of daily living. Gerontologist 1969; 9:179-85.

31. Ministério do Trabalho e Emprego. Classificação Brasileira de Ocupações. http://www.mtecbo. gov.br/cbosite/pages/home.jsf (acessado em 25/ Jan/2011).

32. Lee ES, Forthofer RN. Analyzing complex survey data. 2nd Ed. Beverly Hills: Sage; 2006.

33. Cruickshanks KJ, Wiley TJ, Tweed T, Klein BEK, Klein R, Mares-Perlman JA, et al. Prevalence of hearing loss in older adults in Beaver Dam, Wisconsin: the epidemiology of hearing loss study. Am J Epidemiol 1998; 148:879-86.

34. Coelho Filho JM, Ramos LR. Epidemiologia do envelhecimento no Nordeste do Brasil: resultados de inquérito domiciliar. Rev Saúde Pública 1999; 33:445-53.

35. Desai M, Pratt L, Lentzner H, Robinson K. Trends in vision and hearing among older Americans. Aging Trends 2001; (2):1-8.

36. Sindhusake D, Mitchell P, Smith W, Golding M, Newall P, Hartley D, et al. Validation of self-reported hearing loss: the Blue Montains Hearing Study. Int J Epidemiol 2001; 30:1371-8.

37. Liu C, Xing G, Xu X, Chen Z, Zhou H, Wang D, et al. The application of improved CHQS for mass epidemiology study on hearing impairment. Lin Chung Er Bi Yan Hou Tou Jing Wai Ke Za Zhi 2010; 24:19-20. 
38. Castillo E, Carricondo F, Bartolomé MV, VicenteTorres A, Broto JP, Gil-Loyzaga P. Presbiacusia: degeneración neuronal y envejecimiento en el receptor auditivo del ratón C57/BL6J. Acta Otorrinolaringol Esp 2006; 57:383-7.

39. Morettin M, Cardoso MRA, Lebrão ML, Duarte YAO. Fatores relacionados à autopercepção da audição entre idosos do município de São Paulo - Projeto SABE. Saúde Coletiva (Barueri) 2008; (5):168-72.

40. Prado AC. Principais características da produção vocal do deficiente auditivo. Rev CEFAC 2007; 9:404-10.

41. Sousa CS, Castro Júnior N, Larsson EJ, Ching TH. Estudo de fatores de risco para presbiacusia em indivíduos de classe socioeconômica média. Braz J Otorhinolaryngol 2009; 75:530-6.

42. Costa LB, Koyama MAA, Minuci EG, Fischer FM. Morbidade declarada e condições de trabalho: o caso dos motoristas de São Paulo e Belo Horizonte. São Paulo Perspect 2003; 17:7-11.

43. AgrawalY, Platz EA, Niparko JK. Prevalence of hearing loss and differences by demographic characteristics among US adults. Arch Intern Med 2008; 168:1522-30.

44. McFadden D. A speculation about the parallel ear asymmetries and sex differences in hearing sensitivity and otoacoustic emissions. Hear Res 1993;68:143-51.

45. Copeman WSC. Rheumatoid oto-arthritis? BMJ 1963; 2:1526-7.
46. Stone JH, Francis HW. Immune mediated inner ear disease. Curr Opin Rheumatol 2000; 12:32-40.

47. Gopinath B, McMahon CM, Rochtchina E, Mitchell P. Dizziness and vertigo in an older population: the Blue Mountains prospective cross-sectional study. Clin Otolaryngol 2009; 34:552-6.

48. Ganança MM, Caovilla HH, Ganança F. Como lidar com a vertigem no idoso. São Paulo: Janssen-alg; 1996.

49. Gushikem P, Caovilla HH, Ganança MM. Avaliação otoneurológica em idosos com tontura. Acta ORL 2003; 21:7-11.

50. Klein BE, Cruickshanks KJ, Nondahl DM, Klein R, Dalton DS. Cataract and hearing loss in a population-based study: the Beaver Dam studies. Am J Ophthalmol 2001; 132:537-43.

51. Veiga LR, Merlo ÁRC, Mengue SS. Satisfação com a prótese auditiva na vida diária em usuários do Sistema de Saúde do Exército. Rev Bras Otorrinolaringol 2005; 71:67-73.

52. Northern JL. Pacient satisfaction and hearing aid outcomes. Hear J 2000; 53:10-6.

53. Veras RP, Caldas CP. Promovendo a saúde e a cidadania do idoso: o movimento das universidades da terceira idade. Ciênc Saúde Coletiva 2004; 9: 423-32.

Recebido em 16/Jan/2012

Versão final reapresentada em 02/Abr/2012

Aprovado em 17/Abr/2012 